\title{
THE VUV/UV OK-5 DUKE STORAGE RING FEL WITH VARIABLE POLARIZATION*
}

\author{
O.A. Shevchenko ${ }^{\dagger}$, V.N. Litvinenko, S.M. Mikhailov, FELL, Duke University, Durham, NC 27708, \\ USA \\ N.A. Vinokurov, N.G. Gavrilov, P.D. Vobly, BINP, Novosibirsk, 630090, Russia \\ Y. Wu, LBNL, Berkeley, CA 94720, USA
}

\begin{abstract}
We discuss the design and expected performance of the OK-5 FEL, which will replace in 2002 the OK-4 FEL operating now at the Duke storage ring. The OK-5 FEL is the first distributed optical klystron with variable polarization. The design of wigglers and matching sections is flexible for providing optimal conditions for variety of operation modes. In this paper we present calculations of the main OK-5 FEL parameters such as its tuning range, gain and lasing power. Based on our calculations we present the predictions for the OK-5 gamma-ray source. We conclude with description of the current status of the OK-5 FEL and plans for its installation.
\end{abstract}

\section{INTRODUCTION}

The OK-5/Duke storage ring FEL project was started in 1998. Presently, the components of the OK-5 FEL and the new South straight section of the storage ring are in the final stage of manufacturing. The OK-5 FEL will become a logical continuation of the series of optical klystrons (OK 1 - 4), which were initially developed in the Budker Institute of Nuclear Physics, Novosibirsk [1]. We expect that the OK-5 will replace the OK-4 FEL at the Duke storage in 2002.

The principal feature of the OK-5 is its controllable polarization, which will enable us to carry out a number of new experiments. They include some nuclear physics experiments with polarized targets, studies of the dichroizm of biological objects and the experiment on the parity violation in atomic transitions $1 \mathrm{~S}-2 \mathrm{~S}$ in hydrogen.

The choice of the polarization includes but is not limited to the left and right circular polarization, and the horizontal and the vertical linear polarization. However the main mode of the OK-5 FEL will be based on the circular polarization. We plan to use the elliptical polarization of the wigglers for all OK-5 FEL operations. To lase with linear polarization we will use the opposite helicity of the adjacent wigglers. The use of the elliptical polarization will provide for strong reduction of the

\footnotetext{
* Work supported Office of Naval Research Grant \#N00014-94-1-0818, by the Duke University School of Arts and Sciences, by the Director, Office of Energy Research, Office of Basic Energy Sciences, Material Sciences Division, U.S. Department of Energy, under Contract No. DEAC03-76SF00098

'shevchen@fel.duke.edu
}

thermal load and the degradation of the downstream mirror compared with the straightforward use of plane wiggler field.In Sec. 2 we briefly describe the OK-5 lattice design, we also present the results for the OK-5 FEL gain, lasing power and $\gamma$-ray beam parameters. Sec. 3 is dedicated to the present status of the project and future plans.

\section{DESIGN AND EXPECTED PERFORMANCE OF THE OK-5}

\subsection{General design.}

The detailed description on the OK-5 Duke storage ring FEL and its lattice design can be found at [2-4]. The schematic layout of the OK-5 FEL system is shown in Fig. 1. The 24.2-meter long magnetic system of the OK-5 comprises four electromagnetic wigglers with controllable polarization and three midsections between them. The length of the segment (the wiggler + midsection) is equal to $1 / 8$ of the ring circumference. This configuration provides for effective generation of $\gamma$-rays using 8 ebunches and three collision points. Each mid-section has a buncher and a triplet of qudrupoles. The OK-5 magnetic system fits into the center of the 34.2-meter long, dispersion free South straight section of the Duke storage ring. Two 5-m long bilaterally symmetric sections with four quadrupoles provide the matching between the arcs and the OK-5 system. The lattice of the OK-5 is very flexible providing the tunability of the $\beta$-functions in center of each wiggler from $4 \mathrm{~m}$ to $10 \mathrm{~m}$ in both directions.

The main components of the OK-5 FEL are four electromagnetic wigglers (EM) with controllable polarization. The main advantages of the helical wigglers are the increase of the gain (almost by a factor of two at fixed period and wavelength) and the absence of high harmonics of radiation on its axis. The helical wiggler is composed of two planar wigglers shifted with respect to each other on a quarter of the wiggler period. Each component of the field is independently controllable by the coil current in vertical and horizontal arrays. The 4meter quarter-yokes of the wiggler are made from soft magnetic steel on high precision milling machine. The pole tips are made independently and pinned to the yokes. The final accuracy of the assembly is about 20 microns. 


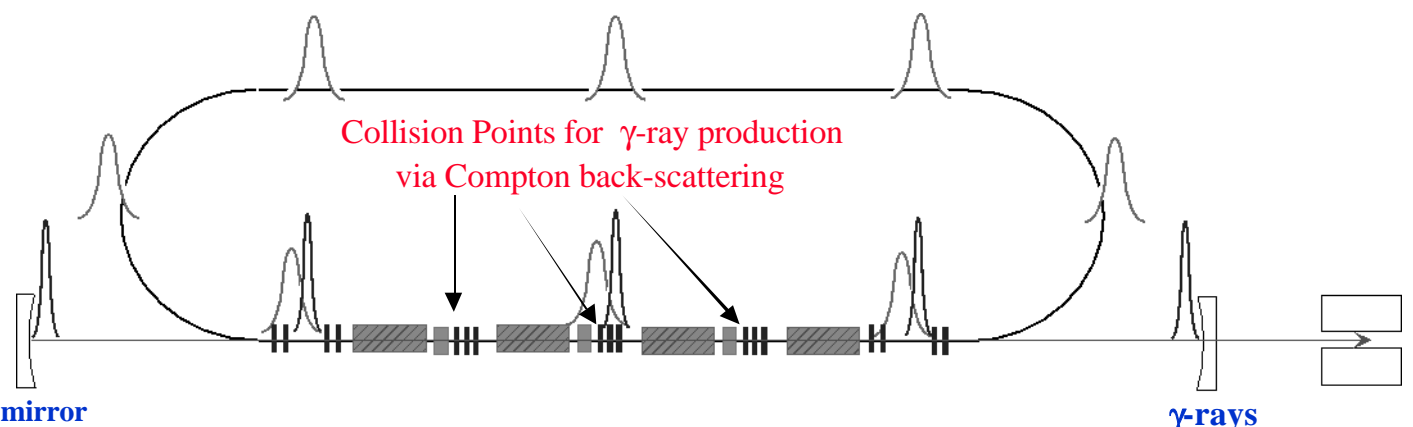

Cavity mirror

OK-5 FEL

Figure 1. Schematic layout of the OK-5/Duke storage ring FEL and $\gamma$-ray source.

The requirement for switching the helicity of radiation leads to violation of the helical symmetry of the wiggler design. As the result, the additional steps should be taken to compensate asymmetry of the magnetic field. Special design of the wiggler terminations is also required to provide for the adiabatic entrance of particles into the regular part of the wiggler.

\subsection{Expected parameters}

The wavelength of the OK-5 FEL spontaneous radiation can be tuned from $11 \mathrm{~nm}$ to $2.3 \mu \mathrm{m}$ using ebeam energy from $0.3 \mathrm{GeV}$ to $1.2 \mathrm{GeV}$. The OK-5 tuning range is shown in Fig.2. The lasing range will be limited by the OK-5 FEL gain and the reflectivity of the available mirrors. We expect to extend the lasing with OK-5 FEL installed on the existing Duke storage ring down to $100 \mathrm{~nm}$ or even lower.

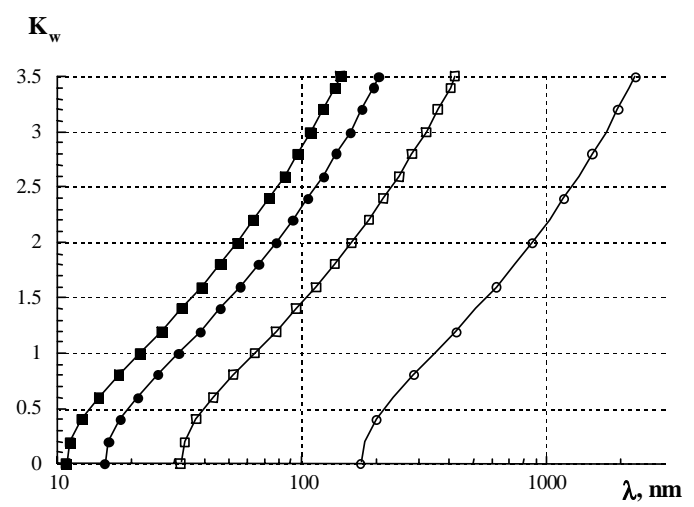

Figure 2. Tuning range of the spontaneous radiation of the OK-5 helical wigglers. $\mathrm{O}-\mathrm{E}=0.3 \mathrm{GeV} ; \square-\mathrm{E}=$ $0.7 \mathrm{GeV}, \bullet-\mathrm{E}=1 \mathrm{GeV}$, and $\boldsymbol{\square}-\mathrm{E}=1.2 \mathrm{GeV}$

We used RON [5] to calculate the dependencies of the OK-5 FEL gain on the e-beam energy, emittances and energy spread. We focused on the short wavelengths to explore the limits of the OK-5 FEL performance. The OK- 5 has larger gains in the visible and the near-IR ranges. Two typical dependencies of the OK-5 FEL gain (at 100 and $200 \mathrm{~nm}$ ) on the beam current are shown in the Fig. 3.

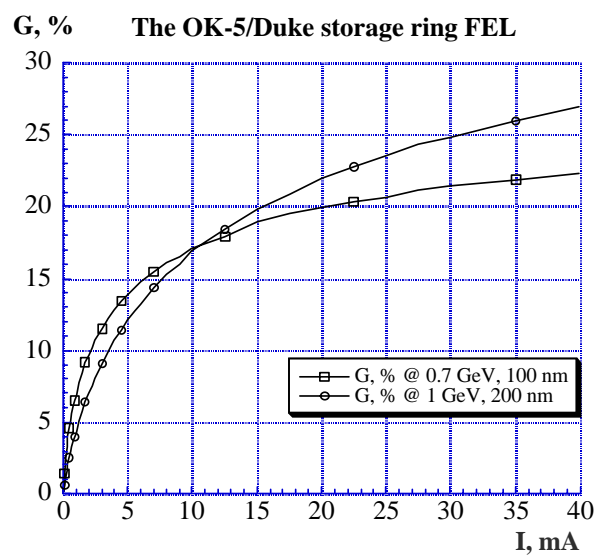

Figure 3. The calculated OK-5 gain as a function of the bunch current. $\mathrm{V}_{\mathrm{rf}}=700 \mathrm{kV}$, peak current is $\mathrm{I}_{\text {peak }}=40.3$ A for $\mathrm{I}=20 \mathrm{~mA} / \mathrm{bunch}\left(\mathrm{O}-\lambda=200 \mathrm{~nm}, \mathrm{E}=1 \mathrm{GeV}, \varepsilon_{\mathrm{x}}\right.$ $=19 \mathrm{nmrad}, \varepsilon_{\mathrm{y}}=1.9 \mathrm{nmrad} ; \square-\lambda=100 \mathrm{~nm}, \mathrm{E}=0.7$

$\left.\mathrm{GeV}, \varepsilon_{\mathrm{x}}=10 \mathrm{nmrad}, \varepsilon_{\mathrm{y}}=1.0 \mathrm{nmrad}\right)$.

Our predictions for the lasing power are based on the approximate formula for the lasing power of an OK installed on the storage ring [6]. This formula is in a good agreement with the experimental results obtained for the OK-4/Duke storage ring FEL operated in both the OK and the conventional FEL configurations [6]. The optimized average power of storage ring FEL is:

$$
P_{\text {lasing }} \cong \xi_{E} P_{S R} \frac{\sigma_{E \text { lasing }}^{2}-\sigma_{E \mathrm{o}}^{2}}{E \cdot \sigma_{E \text { lasing }}} e^{-1 / 2},
$$

where $\mathrm{P}_{\mathrm{SR}}$ is the power of the synchrotron radiation, $\sigma_{\mathrm{E} \text { lasing }}$ is the steady-state electron beam energy spread in the $\mathrm{CW}$ lasing mode. The rest of parameters are specified in ref. [6] and Table I. Table I provides specific example of the lasing power calculated for the OK-5 FEL. The OK-5 FEL will induce $\sigma_{\mathrm{E} \text { lasing }} \sim 0.3 \%$, which is sufficient for complete suppression of the 
microwave instability (MWI). With the lasing, the peak e-beam current will be $\sim 5-10$ times lower than the threshold of MWI, which makes the formula (1) valid. To estimate the OK-5 lasing power, we used RON for calculating the OK-5 FEL gain dependence on the ebeam energy spread. From these dependencies we found $\sigma_{\text {Elasing }}$ by setting the OK-5 FEL gain to be equal the round trip loss in the optical cavity, i.e. a steady state lasing. Table I summarizes a specific case for the deep-UV $0.9-1.0 \mathrm{GeV}$ operation of the OK-5 FEL.

Table 1: Example of the lasing power and $\gamma$-ray flux for OK-5 FEL

\begin{tabular}{|c|c|c|}
\hline Wavelength, $\mathrm{nm}$ & $200-240$ & \\
\hline Beam energy, GeV & $0.9-1.0$ & \\
\hline Beam current in 8 bunches, $\mathrm{mA}$ & 160 & \\
\hline $\mathrm{P}_{\mathrm{SR}}, \mathrm{kW}$ & $4.4-6.7$ & \\
\hline Energy partition number, $\xi_{\mathrm{s}}$ & 2.07 & \\
\hline Natural energy spread, $\sigma_{\mathrm{E},}, \%$ & $0.052-$ & \\
& 0.058 & \\
\hline Mode of operation & FEL users & $\gamma$-rays \\
\hline Total optical cavity loss, $\%$ & 2.1 & 1.1 \\
\hline Mirrors transparency, $\%$ & 1.1 & 0.1 \\
\hline Lasing energy spread, $\sigma_{\mathrm{E} \text { lasing }} \%$ & 0.288 & 0.374 \\
\hline Average lasing power, $\mathrm{W}$ & $15-23$ & $20-31$ \\
\hline Power delivered to users, $\mathrm{W}$ & $8-12$ & $1.8-2.8$ \\
\hline Average intracavity power, $\mathrm{kW}$ & & $60-87$ \\
\hline Peak energy of $\gamma$-rays, MeV & & \\
\hline Average flux, $\gamma$-rays per second & & $2.8-$ \\
& & $4.2 \cdot 10^{10}$ \\
\hline
\end{tabular}

\section{CONCLUSION}

The OK-5 FEL/Duke storage ring FEL promises further advanced of FEL-oscillators into the UV and VUV range. It has substantial gain to operate below $200 \mathrm{~nm}$. It will provide remarkable features for the users of spontaneous and coherent radiation in the UV and the VUV ranges of spectra. The real potential of the OK-5 FEL will be realized after modification of the Duke storage ring with lower emittance and low impedance vacuum chamber. Gain $\sim 1,000 \%$ per pass will allow the OK-5 to lase below $100 \mathrm{~nm}$ and to generate coherent soft-X-ray harmonics. The final design of all components of the OK-5 FEL and 34-m straight sections was completed by mid-2000. Four OK-5 wigglers have been manufactured and are undergoing magnetic measurements, fine tuning and minor modification of the pole-tips. The quality of the wigglers seems to be excellent. The rest of the system is in the production stage and should be finished in the summer of 2001. The OK-5 FEL system will go through additional tests at Duke University prior to installation onto the Duke storage ring. We hope to report the first results attained with the OK-5/Duke storage ring in 2002.

\section{REFERENCES}

[1] G.A.Kornyukhin, G.N.Kulipanov, V.N.Litvinenko, N.A.Mezentsev, A.N.Skrinsky, N.A.Vinokurov and P.D.Vobly, Nucl. Instr. and Meth. A237 (1985) 281.

[2] V.N.Litvinenko, S.F.Mikhailov, N.A.Vinokurov, N.G.Gavrilov, G.N.Kulipanov, O.A.Shevchenko, P.D.Vobly, "Helical wigglers for the OK-5 storage ring VUV FEL at Duke", accepted for publication in Nucl. Instr. and Meth.

[3] Y. Wu, V. N. Litvinenko, S. F. Mikhailov, N. A. Vinokurov, N.G. Gavrilov, O.A.Shevchenko, T.V.Shaftan, D.A.Kairan, "Lattice Modification and Nonlinear Dynamics for Elliptically Polarized VUV OK-5 FEL Source at Duke Storage Ring", accepted for publication in Nucl. Instr. and Meth.

[4] V.N.Litvinenko, S.F.Mikhailov, N.A.Vinokurov, N.G.Gavrilov, D.A.Kairan, G.N.Kulipanov, O.A.Shevchenko, T.V.Shaftan, P.D.Vobly, Y. Wu, "The OK-5/Duke Storage Ring VUV FEL with Variable Polarization", accepted for publication in Nucl. Instr. and Meth.

[5] R.J.Dejus, O.A.Shevchenko, N.A.Vinokurov, Nucl. Instr. and Meth A 445 (2000) 19

[6] V.N.Litvinenko, S.H.Park, I.V.Pinayev, Y.Wu, "Average and Peak Power Limitations and Beam Dynamics in the OK-4/Duke storage ring FEL" accepted for publication in Nucl. Instr. and Meth. 